\title{
Gingival Overgrowth in Subjects under Immunosuppressive Regimens Based on Tacrolimus or Combination of Tacrolimus and Amlodipine
}

\section{Fatima Hassan, Nada Tawfig* and Bakri Gobara}

Department of Periodontology, Faculty of Dentistry, University of Khartoum, Khartoum, Sudan

\begin{abstract}
Background: Tacrolimus is a new-generation immunosuppressant as successful as cyclosporine in suppressing organ transplant rejection and recent reports suggest patients taking it complain in frequently of gingival problems.

Objectives: This study aimed to determine prevalence of gingival overgrowth among renal transplant recipients (RTRs) and to compare its occurrence in patients who received only tacrolimus and those who were on tacrolimus and amlodipine.
\end{abstract}

Methods: RTRs were recruited from 3 Kidney Transplant Units (Khartoum, Sudan). Participants completed a standardized questionnaire and received periodontal examination, including the vertical gingival overgrowth index (GOi) and the simplified-oral hygiene index (OHI-S)

Results: Among 308 RTRs, 29 (9.8\%) presented with gingival overgrowth, with the highest prevalence among those taking tacrolimus and amlodipine while those taking tacrolimus alone did not show gingival overgrowth. Tacrolimus was not found to be associated with GE. Poor oral hygiene was significantly observed on those with gingival overgrowth and took a combination of amlodipine and tacrolimus.

Conclusions: There is a strong correlation between gingival overgrowth, and combination therapy with tacrolimus and amlodipine in transplant patients compared to those treated by tacroloimus alone. Amlodipine reduction may restrain this adverse effect.

Keywords: Amlodipine, Tacrolimus, Gingival overgrowth

\section{Introduction}

It is well known that kidneys perform four essential functions in human body. These are excretion of the end products of metabolism, particularly urea, regulation of blood volume and electrolyte concentration, regulation of erythrocyte production in the bone marrow through the secretion of erythropoietin and participation in calcium homeostasis through hydroxylation of vitamin D3 into active or inactive metabolites. Therefore, any pathologic process that results in decreased renal function would be expected to have serious effects [1]. Many diseases affect the kidney. One of them is chronic kidney disease (CKD), which is an abnormality in kidney structure or function and is defined as a reduced glomerular filtration rate, increased urinary excretion or both [2,3]. Chronic kidney disease has become a major public health problem due to its high prevalence which is estimated to be $8-16 \%$ worldwide [3,4], large reductions in life expectancy and quality of life of affected people. On the other hand studies have shown that the prevalence of chronic kidney disease in Sudan ranged between $4-24 \%[3,5]$. Seventy percent of cases progress to end-stage renal disease (ESRD) due to diabetes and hypertension, which contributes to progression of kidney disease towards end stage [6,7]. In Sudan, the cause of ESRD for those who were younger than 40 years was glomerular disease, hypertension for those between 40 and 60 years and obstruction for those who were older than 60 years [8].

End stage renal disease is fatal without renal replacement therapy. Patients with end stage renal disease are treated by renal transplantation, which is the preferred form of renal replacement therapy $[1,9,10]$. Continuous immune suppression after renal transplantation prevents graft rejection, as it inhibits cellular, humoral or both the immune responses. Cyclosporine and tacrolimus are highly effective drugs and both comprise the backbone of transplant immune-suppression $[11,12]$.

Despite the fact that cyclosporine has a great effect in preventing graft rejection, it was associated with a variety of side effects, including nephrotoxicity, hypertension, diabetes, dyslipidemia, neurotoxicity, osteopenia and oral changes such as gingival overgrowth $[13,14]$.

Many studies have shown that gingival overgrowth is commonly found in immunosuppressive regimens based on Cyclosporine A. Several factors other than cyclosporine use, such as the use of prednisone, azathioprine, and calcium channel blockers (CCB), as well as gingival inflammation, appears to have an influence on the occurrence and severity of this event $[15,16]$.

Tacrolimus has been used successfully to prevent acute rejection of renal transplantation. It inhibits calcineurin, a $\mathrm{Ca}^{2+}$ calmodulindependent phosphatase, which results in reduced expression of interleukin- 2 by T lymphocytes [17]. Tacrolimus has been associated with many side effects including nephrotoxicity, neurotoxicity and the induction of a diabetic state. Unlike cyclosporine A, studies that evaluated immunosuppressive agents based on tacrolimus failed to demonstrate gingival overgrowth within tacrolimus-based therapies $[16,18]$, while others demonstrated a lower prevalence and severity of gingival overgrowth when compared with cyclosporine A-based therapies $[15,19]$.

There is scarce data among Sudanese population related to comparisons of prevalence and severity of gingival overgrowth within

*Corresponding author: Nada Tawfig, Assistant Professor, Department of Periodontology, Faculty of Dentistry, University of Khartoum, Khartoum, Sudan, Tel: 0024 9912347374; E-mail: nadatawfig@yahoo.com

Received August 10, 2015; Accepted August 24, 2015; Published September 04, 2015

Citation: Hassan F, Tawfig N, Gobara B (2015) Gingival Overgrowth in Subjects under Immunosuppressive Regimens Based on Tacrolimus or Combination of Tacrolimus and Amlodipine. Dentistry 5: 331. doi:10.4172/2161-1122.1000331

Copyright: $\odot 2015$ Hassan F, et al. This is an open-access article distributed under the terms of the Creative Commons Attribution License, which permits unrestricted use, distribution, and reproduction in any medium, provided the original author and source are credited. 
immunosuppressive regimens. Therefore, the purpose of the present study was to evaluate the prevalence and severity of gingival overgrowth among renal transplant recipients under immunosuppressive regimens based, tacrolimus, and combination of tacrolimus and amlodipine.

\section{Materials and Methods}

\section{Study design and sampling}

The present study used a cross-sectional design and involved a subset of renal transplant recipients (RTRs) under immunosuppressive maintenance therapies from Khartoum city, Sudan. It was approved by Research Committee, faculty of Dentistry, University of Khartoum. A letter was sent to the authorities in Ahmed Gasim Hospital, Ibn Sina Specialized Hospital and Sharg El Neel Hospital prior to the conduction of the study. A written permission was obtained from the directors of the three hospitals.

All participants were informed of the goals of the study and provided a written informed consent before their participation in the study. Subjects' rights were protected at all times.

\section{Study population and recruitment strategies}

Adult RTRs were recruited from the outpatient Kidney Transplant Unit at Ahmed Gasim Hospital, Ibn Sina Specialized Hospital, and Sharg El Neel Hospital, between August and December 2014 according to the following inclusion criteria,

a) willingness of the patient to participate in the study and able to undergo a comprehensive oral and periodontal examination,

b) adult patients taking tacrolimus,

c) adult patients taking tacrolimus and amlodipine.

Subjects were recruited by a researcher, who explained the study objectives, obtained written informed consent, and interviewed each participant to complete a questionnaire.

For the present analysis the following subjects were excluded

a) pregnant women,

b) patients with history of hypertension before renal transplantation,

c) patients taking other drugs which cause gingival overgrowth,

d) patients who had a history of taking cyclosporine drugs after renal transplant.

\section{Periodontal examination}

All patients received a comprehensive periodontal examination. The examiner was calibrated prior to the study with an agreement rate for the intraexaminer reliability $\ddagger 0.85$ for the full-mouth periodontal measurements. The examiner masked the participants' medication regimen. A full-mouth periodontal examination was performed on all teeth.

Clinical assessments included Clinical evaluation of the patients was implemented using the vertical gingival overgrowth index (GOi) $[16,20]$ and the simplified-oral hygiene index (OHI-S) $[21,22]$. Both indices were measured for all teeth present using William's pattern probes. GOi was measured vertically in the apex-crown direction from the cemento-enamel line to the free gingival margin at six sites around each tooth [20]. OHI-S was used to assess the oral hygiene of patients which consisted of two components (debris index-simplified and calculus index-simplified) [23]. Four posterior and two anterior teeth were examined [24]. Posteriorly, the buccal surfaces of the upper molars and the lingual surfaces of the lower molars were scored. While anteriorly, the labial surfaces of the upper right and the lower left central incisors were scored.

The scores for debris and calculus index were recorded, calculated and then added to obtain the simplified-oral hygiene index, which was scored from 0 to 6 [25].

\section{Questionnaire data}

Data was collected using interview questionnaires and examination sheets. The questionnaire was filled by the examiner by directly interviewing renal transplant adult patients. It was composed of two domains. The first domain was for the patient's personal information including, age, gender, residence and the telephone number.

The second domain covered the medical history of the patient including, systemic conditions, date of diagnosing renal failure, date of dialysis and kidney transplant, as well as the dose of the tacrolimus and amlodipine.

\section{Statistical Analysis}

Data was checked for consistency and completeness and was analysed using Statistical Package for Social Sciences (IBM SPSS) version 20. Descriptive analysis was done for the variables such as the drug dose, duration of drug, time since diagnosis, time since dialysis, age, gender and residence. Differences in proportions were tested using the Chi-square test. Independent-samples T-tests were done for the clinical parameters. p-value $\leq 0.05$ was considered statistically significant. Data was displayed in appropriate tables and figures format using Excel and Microsoft document. The inter-rater reliability analysis using the Kappa statistic was performed to determine consistency among raters.

\section{Results}

\section{Sample characteristics and clinical parameters}

A total of 308 adult RTRs enrolled in this study and underwent a complete periodontal examination. The participants were divided into 2 groups according to medication regimen. Group A consisted of 147 subjects $(47.7 \%)$ who were taking a regimen that included tacrolimus. Group B consisted of 161 subjects (52.3\%) whose regimen included a combination of tacrolimus and amlodipine (Figure 1).

The age of patients involved in the study ranged between 20 to 60 years and above. In the tacrolimus group the mean and standard deviation of age was $(40.9 \pm 13.1)$ years, whereas in the tacrolimus with amlodipine group was $(43.3 \pm 13.8)$ years.

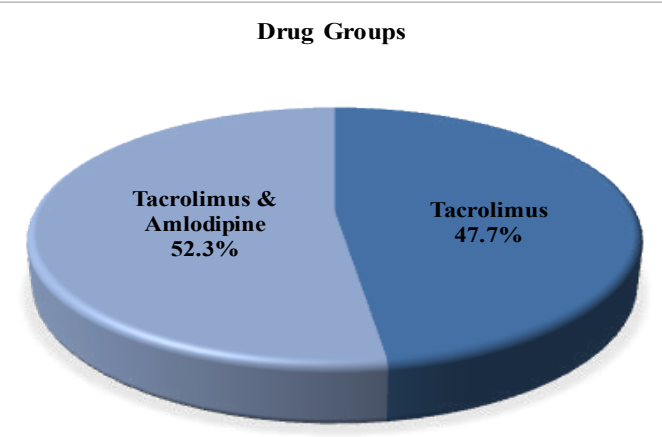

Figure 1: Proportions of tacrolimus patients and tacrolimus with amlodipine group of patients $(\mathrm{N}=308)$ 
All patients with kidney transplant were on steroids (100\%). Patients taking amlodipine due to hypertension were almost 161 (52.3\%). Azathioprine (Imuran) was taken by 203 patients $(65.9 \%)$, while 53 (17.2\%) were taking mycophenolate sodium (Myfortic) and 39 (12.7\%) were taking mycophenolate mofetil (Cellcept). 13 (4.2\%) were found to be diabetic and on insulin treatment. Patients taking septrin were $30(9.7 \%)$, while ranitidine was taken by $36(11.7 \%)$ patients and 4 (1.3\%) were taking fefol. Lisinopril, bisoprolol, atenolol, doxazosin and acyclovir taken by less than $0.6 \%$ patients

Using OHI-S, results showed 139 (45.1\%) participants had good oral hygiene, while 121 (39.9\%) had fair oral hygiene, whereas $48(15.6 \%)$ had poor oral hygiene. The majority of patients taking tacrolimus have good oral hygiene (61.9\%), while the majority of those taking tacrolimus and amlodipine have fair oral hygiene $(51.6 \%)$ $(\mathrm{p}=0.001)$ (Table 1).

Overall, 29 of 308 subjects (9.4\%) presented with gingival overgrowth. It was not observed among patients taking Tacrolimus, while it was found in $29(18 \%)$ patients taking tacrolimus and amlodipine. There was a statistically significant association between gingival overgrowth grade and type of drugs used, $\mathrm{p}=0.001$ (Table 2). 20 (66.7\%) patients with gingival overgrowth had poor oral hygiene which was found to be statistically significant $(\mathrm{p}=0.001)$ (Table 3 ).

\section{Discussion}

The prevalence of gingival overgrowth in this group of 308 renal transplant patients was $9.4 \%$ overall, with the highest figure among those taking a combination of tacrolimus and amlodipine. The renal transplant patients taking only tacrolimus did not show any sign of gingival overgrowth. This result is consistent with that of several previous studies $[18,26]$.

Previous studies have shown that the use of calcium channel blockers alone or in combination with Cyclosporine or Tacrolimus is related to an increased prevalence and severity of GO [26,27]. Ellis et al. reported that gingival overgrowth scores in the Tacrolimus group were greater when they were medicated with calcium channel blockers, this indicates that the use of CCBs presented a great influence on the occurrence and severity of gingival overgrowth [26].

The role of the dental biofilm in the etiology of gingival overgrowth has not been well elucidated. Study by Daley et al. showed that the presence of a biofilm was associated with gingival overgrowth, but the relationship between the amount of plaque and the severity of gingival overgrowth is not assured [28]. Furthermore McGaw et al. observed a higher bacterial PI in patients with gingival overgrowth [29]. In this study, poor oral hygiene was found on a significantly larger proportion of sites among RTRs with gingival overgrowth than among those without it. This finding is in agreement with previous findings $[15,30,31]$ and this may be a clue to consider plaque as an important co-factor in the etiology of drug associated gingival enlargement [32].

With respect to duration of tacrolimus intake, after renal transplantation patients take high doses of tacrolimus ranging from $8-11 \mathrm{mg} / \mathrm{dl}$, within years the dose is lowered till it reaches $1-2 \mathrm{mg} /$ dl. Since the present study was started after lowering the dose for all transplanted patients taking tacrolimus, the duration of the drug was not consider as a variable in the study.

\section{Conclusions}

Tacrolimus is not associated with gingival overgrowth. If possible, calcium channel blockers should be avoided by those patients taking

\begin{tabular}{|l|l|l|l|l|l|}
\hline \multirow{2}{*}{ Drug } & \multicolumn{5}{|l|}{ Oral Hygiene Status } \\
\cline { 4 - 6 } & & Good & Fair & Poor & Total \\
\hline \multirow{3}{*}{ Tacrolimus } & Number of Patients & 91 & 38 & 18 & 147 \\
\hline Percentage & $61.9 \%$ & $25.9 \%$ & $12.2 \%$ & $100.0 \%$ \\
\hline $\begin{array}{l}\text { Tacrolimus \& } \\
\text { Amlodipine }\end{array}$ & Number of Patients & 48 & 83 & 30 & 161 \\
\hline \multirow{2}{*}{ Total } & Percentage & $29.8 \%$ & $51.6 \%$ & $18.6 \%$ & $100.0 \%$ \\
\hline & Number of Patients & 139 & 121 & 48 & 308 \\
\cline { 2 - 6 } & Percentage & $45.1 \%$ & $39.3 \%$ & $15.6 \%$ & $100.0 \%$ \\
\hline
\end{tabular}

${ }^{*}$ Chi-square test was performed, $p$-value $=0.001$ which is significant.

Table 1: Relationship between type of drug and oral hygiene status using $\mathrm{OHI}-\mathrm{S}$ $(\mathrm{N}=308)$.

\begin{tabular}{|l|l|l|l|}
\hline \multirow{2}{*}{ Drug } & \multicolumn{3}{l|}{ Gingival Overgrowth } \\
\cline { 2 - 3 } & Present & Not present & Total \\
\hline Tacrolimus & 0 & 147 & 147 \\
\hline & $0.0 \%$ & $100.0 \%$ & $100.0 \%$ \\
\hline Tacrolimus \& Amlodipine & 29 & 132 & 161 \\
\hline & $18.0 \%$ & $82.0 \%$ & $100.0 \%$ \\
\hline Total & 29 & 279 & 308 \\
\hline & $9.4 \%$ & $90.6 \%$ & $100.0 \%$ \\
\hline
\end{tabular}

${ }^{*}$ Chi square test was performed, $p$-value $=0.001$ which is significant.

Table 2: Relationship between type of drug and gingival overgrowth using GOi.

\begin{tabular}{|l|l|l|l|}
\hline \multirow{2}{*}{ Oral Hygiene Status } & \multicolumn{3}{|c|}{ Gingival Overgrowth } \\
\cline { 2 - 4 } & Present & Not present & Total \\
\hline Good & 1 & 47 & 48 \\
\hline Fair & $2.1 \%$ & $97.9 \%$ & $100.0 \%$ \\
\hline & 8 & 75 & 83 \\
\hline Poor & $9.6 \%$ & $90.4 \%$ & $100.0 \%$ \\
\hline & 20 & 10 & 30 \\
\hline Total & $66.7 \%$ & $33.3 \%$ & $100.0 \%$ \\
\hline & 29 & 132 & 161 \\
\hline & $18.0 \%$ & $82.0 \%$ & $100.0 \%$ \\
\hline
\end{tabular}

${ }^{*}$ Chi square test was performed, $p$-value $=0.001$ which is significant.

Table 3: Relationship between oral hygiene status and gingival overgrowth $(\mathrm{N}=29)$.

tacrolimus or in individuals who do not perform meticulous and good oral hygiene measures.

Patients with chronic kidney disease must be explained about the importance of proper oral hygiene practices and are advised to follow the same.

\section{Limitations}

There are so many potential risk factors such as genetic susceptibility and demographics which contribute gingival overgrowth [33]. A study by Rodwan et al. has shown that HLA-DR2 phenotype was a risk factor for gingival overgrowth; nevertheless, we did not include genetic factors in our survey [34]. Therefore, a large prospective randomized control trial is still required.

\section{References}

1. Craig RG (2008) Interactions between chronic renal disease and periodontal disease. Oral Dis 14: 1-7.

2. Lane BR, Demirjian S, Derweesh IH, Reidinger CB, Fergany AF, et al. (2014) Is all chronic kidney disease created equal? Curr Opin Urol 24: 127-134.

3. Jha V, Garcia-Garcia G, Iseki K, Li Z, Naicker S, et al. (2013) Chronic kidney disease: global dimension and perspectives. Lancet 382: 260-272.

4. Faa G, Sanna A, Gerosa C, Fanni D, Puddu M, Ot, et al. (2015) Renal physiological regenerative medicine to prevent chronic renal failure: Should we start at birth? Clin Chim Acta 444: 156-162. 
Citation: Hassan F, Tawfig N, Gobara B (2015) Gingival Overgrowth in Subjects under Immunosuppressive Regimens Based on Tacrolimus or Combination of Tacrolimus and Amlodipine. Dentistry 5: 331. doi:10.4172/2161-1122.1000331

5. Stanifer JW, Jing B, Tolan S, Helmke N, Mukerjee R, et al. (2014) The epidemiology of chronic kidney disease in sub-Saharan Africa: a systematic review and meta-analysis. The Lancet Global Health 2: 174-181.

6. Toto RD (2005) Treatment of hypertension in chronic kidney disease. Semin Nephrol 25: 435-439.

7. Mallappallil Mary, Friedman Eli A, Delano Barbara G, McFarlane Samy I, Salifu Moro O, et al. (2014) Chronic kidney disease in the elderly: evaluation and management. Clin Pract (Lond) 11: 525-535

8. Elsharif ME, Elsharif EG. (2011) Causes of end-stage renal disease in Sudan: a single-center experience. Saudi J Kidney Dis Transpl 22: 373-376.

9. Bohlke M, Rocha M, Gomes RH, Marini SS, Terhost L, et al. (2006) Tacrolimus and quality of life after kidney transplantation- a multicenter study. Clin Transplant 20: 504-508.

10. Ardalan MR (2014) Global scientific vision with local vigilance: renal transplantation in developing countries. Nephrourol Mon 7: e22653.

11. Ciavarella D, Guiglia R, Campisi G, Di Cosola M, Di Liberto C, et al. (2007) Update on gingival overgrowth by cyclosporine A in renal transplants. Med Oral Patol Oral Cir Buccal 12: 19-25.

12. Danovitch GM (2001) Immunosuppressive medications for renal transplantation: A multiple choice question. Kidney Int 59: 388-402.

13. Nassar CA, Nassar PO, Abi Rached RS, Holzhaushen M, Marcantonio E Jr, et al. (2004) Effect of cyclosporin A on alveolar bone homeostasis in a rat periodontitis model. J Periodontal Res 39: 143-148.

14. Oleg E, Herb S (2011) Essential Immunology for Surgeons. Oxford University Press, London.

15. de Oliveira CF, Diniz FS, de Miranda CLO, Costa JE, Aguiar MA, et al. (2006) Prevalence, severity, and risk variables associated with gingival overgrowth in renal transplant subjects treated under tacrolimus or cyclosporine regimens. J Peridontol 77: 969-975.

16. Greenberg KV, Armitage GC, Shiboski CH (2008) Gingival enlargement among renal transplant recipients in the era of new-generation immunosuppressants. J Periodontol 79: 453-460.

17. Bierer BE, Hollander G, Fruman D, Burakoff SJ (1993) Cyclosporin A and FK506: Molecular mechanisms of immunosuppression and probes for transplantation biology. Curr Opin Immunol 5: 763-773.

18. James JA, Jamal S, Hull PS, Macfarlane TV, Campbell BA, et al. (2001) Tacrolimus is not associated with gingival overgrowth in renal transplant patients. J Clin Periodontol 28: 848-852.

19. Lima RB, Benini V, Sens YA (2008) Gingival overgrowth in renal transplant recipients: a study concerning prevalence, severity, periodontal, and predisposing factors. Transplant Proc 40: 1425-1428.
20. Miranda J, Brunet L, Roset P, Farre M, Mendieta C (2012) Reliability of two measurement indices for gingival enlargement. J Periodontal Res 47: 776-782.

21. Greene JG, Vermillion JR (1964) The simplified Oral Hygiene Index. J Am Dent Assoc 68: 7-13.

22. Podshadley AG, Haley JV (1968) A method for evaluating oral hygiene performance. Public Health Rep 83: 259-264.

23. Reddy S (2014) Essentials of Clinical Periodontology and Periodontics. (4 edn), Jaypee Brothers Medical Publishers, New Delhi.

24. Love WD, Ramirez JM, Fultz RP (1975) An oral hygiene measurement system for possible research and clinical use. J Public Health Dent 35: 227-230.

25. Lemos SS, Oliveira FA, Vencio EF (2010) Peridontal disease and oral hygiene benefits in HIV seropositive and AIDS patients. Med Oral Patol Oral Cir Buccal 15: 17-21.

26. Ellis JS, Seymour RA, Taylor JJ, Thomason JM (2004) Prevalence of gingival overgrowth in transplant patients immunosuppressed with tacrolimus. J Clin Periodontol 31: 126-131.

27. Thomason JM, Seymour RA, Ellis JS (2005) Risk factors for gingival overgrowth in patients medicated with cyclosporine in the absence of calcium channel blockers. J Clin Periodontol 32: 273-279.

28. Daley TD (1986) Clinical and pharmacologic correlations in cyclosporineinduced gingival hyperplasia. Oral Surg Oral Med Oral Pathol 62: 417-421.

29. McGaw T, Lam S, Coates J (1987) Cyclosporine-induced gingival overgrowth: correlation with dental plaque scores, gingivitis scores, and cyclosporine levels in serum and saliva. Oral Surg Oral Med Oral Pathol 64: 293-297.

30. Vescovi P, Meleti M, Manfredi M, Merigo E, Pedrazzi G (2005) Cyclosporineinduced gingival overgrowth: a clinical epidemiological evaluation of 121 Italian renal transplant recipients. J Periodontol 76: 1259-1264.

31. Cezario ES, Cota LO, Ferreira SD, Siqueira FM, Soares RV, Zenobio EG et al. (2008) Gingival overgrowth in renal transplant subjects medicated with tacrolimus in the absence of calcium channel blockers. Transplantation 85: 232-236.

32. Dongari-Baqtzoglou A. (2004) Drug-associated gingival enlargement. J Periodontol 75: 1424-1431

33. Oliveira Costa F, Ferreira SD, Lages EJ, Costa JE, Oliveira AM, et al. (2007) Demographic, pharmacologic, and periodontal variables for gingival overgrowth in subjects medicated with cyclosporine in the absence of calcium channel blockers. J Periodontol 78: 254-261.

34. Radwan-Oczko M, Boratynska M, Klinger M, Zietek M. (2003) Risk factors of gingival overgrowth in kidney transplant recipients treated with cyclosporine $A$. Ann Transplant 8: 57 